\title{
Turbo Coded OFDM Scheme for a High-Speed Power Line Communication
}

\author{
Jin-Young $\mathrm{Kim}^{1}$ and Sung-Wan $\mathrm{Koo}^{1^{*}}$ \\ ${ }^{1}$ Department of Wireless Communication Engineering, Kwangwoon University \\ 고속 전력선 통신을 위한 터보 부호화된 OFDM \\ 김진영 ${ }^{1}$, 구성완 ${ }^{*}$ \\ ${ }^{1}$ 광운대학교 전파공학과
}

\begin{abstract}
In this paper, performance of a turbo-coded OFDM system is analyzed and simulated in a power line communication channel. Since the power line communication system typically operates in a hostile environment, turbo code has been employed to enhance reliability of transmitted data. The performance is evaluated in terms of bit error probability. As turbo decoding algorithms, MAP (maximum a posteriori), Max-Log-MAP, and SOVA (soft decision viterbi output) algorithms are chosen and their performances are compared. From simulation results, it is demonstrated that Max-Log-MAP algorithm is promising in terms of performance and complexity. It is shown that performance is improved $3 \mathrm{~dB}$ by increasing the number of iterations, 2 to 8 , and interleaver length of a turbo encoder, 100 to 5000. The results in this paper can be applied to OFDM-based high-speed power line communication systems.

요 약 본 논문은 전력선 통신 채널에서 터보 부호와 OFDM 시스템의 성능을 분석하고 시뮬레이션 하였다. 전력선 통신 시스템은 최악의 환경에서 동작되기 때문에 터보 부호는 전송 데이터의 신뢰성을 보장하기 위해서 사용되었다. 시뮬레이션의 성능은 비트 오류 확률로써 표현하였다. 터보 복호 알고리즘으로써 MAP과 Max-Log MAP 알고리즘, 그리고 SOVA를 선택하였고, 이것들의 성능을 비교하였다. 시뮬레이션 결과로부터, Max-Log MAP 알고리즘이 성능과 복잡도면에서 유망하다는 것이 입증되었다. 그리고 터보 부호기의 인터리버 길이가 100 에서 5000으로 변할 때와 반 복 횟수가 2 번에서 8 번으로 증가할 때, 각 각 $3 \mathrm{~dB}$ 증가하였음을 알 수 있다. 본 논문에서의 결과는 $\mathrm{OFDM}$ 를 기반으 로 하는 전력선 통신 시스템에 적용될 수 있음을 보여준다.
\end{abstract}

Key Words : OFDM, Power Line, Communication, Turbo Coding.

\section{Introduction}

Power lines originally are designed for delivering power not data. Recently, there is a growing interest on data transmission over a PLC (power line carrier) channel. Undoubtedly, the PLC has considerable potential in many applications such as remote metering, distribution automation, demand-side management, and internet access through home networking. Primary attractions of the PLC are universal presence of electric wiring and ease of access through standardized wall-outlets [1]. However, power lines are heavily affected by interference from various sources and attenuation during transmission exhibits unpredictable variations.

Compared to other communication methods, the PLC systems have the following advantages; 1) wide coverage,

This research was supported by the MKE(The Ministry of Knowledge Economy), Korea, under the ITRC(Information Technology Research Center) support program supervised by the NIPA(National IT Industry Promotion Agency" (NIPA-2009-(C1090-0902-0005))

*Corresponding Author : Koo, Sung-Wan(goodtti@kw.ac.kr)

Received November 10, 2009 Revised (1st December 28, 2009, 2nd January 8, 2010)

Accepted January 20, 2010 
2) modernity, 3) cost effectiveness, 4) easy-to-adoption by consumer, 5) ubiquitousness, and 6) availability worldwide. Unlike other media used for data transmission, which have well defined characteristics for bandwidth, characteristic impedance, and potential noise levels, the PLC is very indeterministic [2-3].

Even allowing for these advantages, these days, the PLC attracts much attention as a means of communication into industrial, commercial, and residential buildings throughout the country [4].

Communication signal in power lines may be corrupted by various electromagnetic radiations, which typically lead to degradation of communication quality. The transmitted signal over the PLC channel is subject to large and unpredictable levels of impedance, noise and attenuation, which vary over time and over network links [5]. Some form of forward error correction scheme is essential to guarantee reliable communications over the PLC channel. So far, in order to improve communication quality, many channel coding schemes such RS (Reed-Solomon) code, convolutional code, and their concatenated code have been proposed for many kinds of communication systems to increase reliability of information transmission.

Since a decade ago, a new error correcting code called 'turbo code' has attracted much attention in the channel coding community. The term turbo is named after the mechanism that the decoder uses its processed output as a priori input in the next iteration. The turbo code can provide significant coding gain by utilizing two constituent convolutional codes and an interleaver. It has been confirmed that the turbo code achieves near Shannon-limit error correcting capability in an AWGN channel. Therefore, it is highly expected that the turbo coding over the power line communication channel lead to considerable coding gain [6-7].

One of promising techniques for high-speed data transmission is OFDM (orthogonal frequency division multiplexing), a kind of multicarrier modulation scheme. The OFDM scheme has the following attractive features for high-speed transmission: 1) high spectral efficiency, 2) robustness in multipath fading environment, 3) low training overhead, and 4) low complexity compared to equalizer, etc. Furthermore, the OFDM scheme has scalability of bit rate and bandwidth in that a sufficient subcarrier bandwidth increases robustness to Doppler spread of a channel. In an OFDM scheme, DFT (Discrete Fourier Transform) is used to multiplex blocks of data symbols over subchannels which are spectrally overlapping yet orthogonal in time [11]. The OFDM has been adopted as a standard for DAB (digital audio broadcasting), WLAN (wireless local area network), and ADSL (asynchronous digital subscriber line).

In this paper, performance of a turbo-coded OFDM system is analyzed and simulated in a power line communication channel. As a modulation scheme, 64-QAM is employed because OFDM/64-QAM is a very strong candidate for high-speed and spectrally-efficient power line communication systems. The performance is evaluated in terms of bit error probability [12]. And, the concepts and principles of turbo code are introduced, and decoding algorithms are described to help readers understand turbo encoding/decoding procedures [14-15].

The simulation results for bit error probability are presented with the following varying parameters: 1) the number of iterations used in the decoding process, 2) interleaver length employed in the turbo encoder, and 3) channel impairment factors of the PLC channel. Finally, the comparative results are shown for the optimal and suboptimal approaches used in the turbo decoding process. For turbo decoding algorithms, the optimal MAP, the suboptimal Max-Log-MAP, and the suboptimal SOVA algorithms are taken into account.

The paper is organized as follows: In Section 2, OFDM/QAM system, PLC channel model, and turbo coding are described. In Section 3, bit error probability for the OFDM/64-QAM system with turbo coding is derived. In section 4, optimal and suboptimal turbo decoding algorithms are described. Simulation results are presented in Section 5, and conclusions are drawn in Section 6.

\section{System Model}

\subsection{OFDM System Model}

In an OFDM system with turbo coding as shown in Fig. 1, input data sequence is first encoded by turbo encoder and then by OFDM/QAM encoder. In the 
OFDM/QAM scheme, a $2^{m-Q A M}$ (quadrature amplitude modulation) is typically used to encode data sequence into phase and magnitude of subcarrier, where $\mathrm{m}$ is the number of bits assigned to the subcarrier. The $\mathrm{m}$ bit groupings of data are encoded as complex values

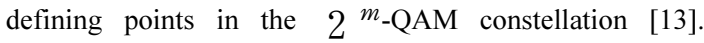
Then, the output of turbo encoder is serial to parallel converted. The OFDM scheme allows spectral overlap of adjacent subcarriers using orthogonal property, which results in high spectral efficiency. The subcarrier frequencies are selected to be spaced at symbol rate. The OFDM modulation and demodulation is efficiently done using FFT and IFFT algorithms. The IFFT output is converted into analog modulating waveform using D/A (digital to analog) converter, and then transmitted.

At the receiver, the recovered baseband signal is sampled and converted to digital form. The FFT is performed to determine phase and amplitude of each subcarrier. For each subcarrier, the transmitted data is estimated through signal point closest to the point corresponding to the received subcarrier. The output of parallel to serial converter is decoded in the turbo decoder to estimate transmitted m-bit data sequence.

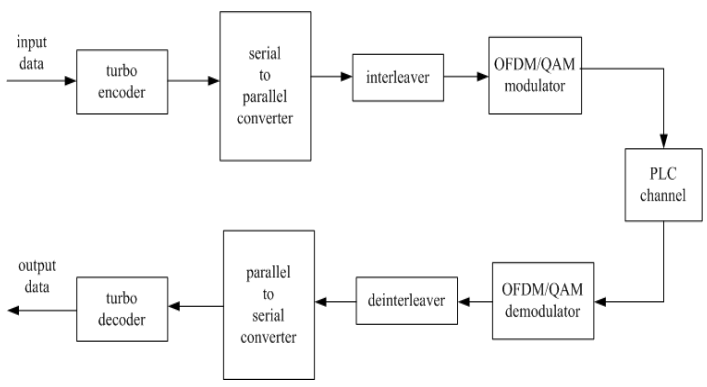

[Fig. 1] Block diagram of a turbo-coded OFDM/QAM system.

\subsection{PLC Channel Model}

Power line can provide reasonably universal channels with a simple and standard interface in the form of a wall socket plug. However, it has disadvantages such as limited bandwidth, high noise levels, varying levels of impedance, attenuation, and noise, etc. Typically, many kinds of loads connected to the power line may act as noise sources. A high and fluctuating noise levels and fluctuating impedance and transmission loss in the power line may incur a considerable problem.

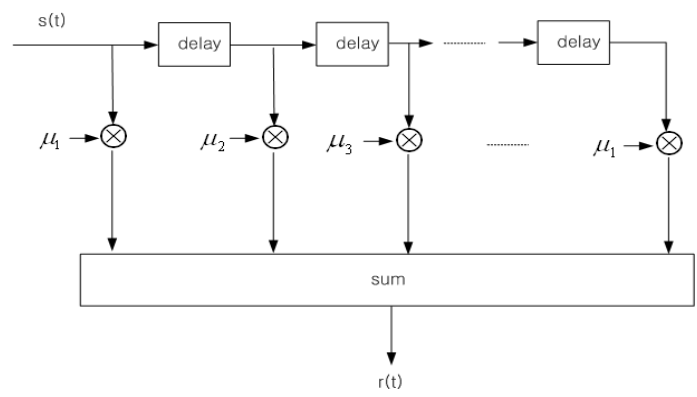

[Fig. 2] PLC channel model.

Since the power line is arranged in the power distribution circuits, the transmission loss is inevitable.

There have been many kinds of channel models for the PLC channel [1]. However, any model cannot completely characterize the PLC channel because various kinds of channel impairment factors are involved in the PLC channel. As shown in Fig. 2, the PLC channel can be modeled as an echo based attenuation model. This echo model has already been confirmed in many kinds of real world environments. Then, its impulse response is given by

$$
h(t)=\sum_{i=1}^{M} \mu_{i} \delta\left(t-\tau_{i}\right),
$$

where $\mathrm{M}$ is the number of echoes in the channel, $\tau_{i}$ and $\mu_{i}$ denote the ith echo delay and attenuation, respectively. In frequency domain, transfer function is given by

$$
H(f)=\sum_{i=1}^{M} \mu_{i} \exp \left(-j 2 \pi f \tau{ }_{i}\right)
$$

Since the attenuation coefficient is a function of cable length and frequency, the ith component can be represented as

$$
\mu_{i}\left(f, l_{i}\right)=\rho_{i} \exp \left(-l_{i} \xi(f)\right),
$$

where $l_{i}$ is cable length, $\rho_{i}$ is a scaling factor 
depending on network topology, and $\xi(f)$ describes a frequency dependent attenuation coefficient. The $\xi(f)$ is involved in skin effect and dielectric loss of insulating material, and is a function of resistance per length, lateral conductivity per length, and characteristic impedance. The simplified approximation of $\xi(f)$ has been verified from numerous measurement results, and is given by

$$
\xi(f)=c_{o}+c_{1} f^{\varepsilon},
$$

where the coefficients $c_{0}, c_{1}$, and $\varepsilon$ is constant for a specific cable type.

\subsection{Turbo Code}

To enhance reliability of information bits, many channel coding schemes such as block and convolutional codes have been proposed [8]. In order to achieve higher coding gains, their concatenated coding scheme has been proposed [9]. In a concatenated coding scheme, probability of error decreases exponentially while decoding complexity increases algebraically. In applications requiring higher coding gain such as deep space communications, the concatenated code has attracted much attention as a powerful coding scheme. The turbo code is a kind of concatenated code which consists of two or more constituent codes. Typically, two recursive systematic convolutional codes are used as the constituent codes [10].

In general, turbo code consists of two or more constituent codes and an interleaver. The first decoder passes the extrinsic information (a part of the soft output provided by a posteriori probability algorithm) to the next decoding stage. For every iteration process, a single decoding is performed using the observation as well as reliability information delivered by the other decoders that were acting before. The MAP (maximum a posteriori) decoding algorithm is known to be an optimal algorithm for turbo decoding process. There are also suboptimal algorithms such as SOVA (soft output Viterbi algorithm) or Max Log MAP that are less complex.

The turbo encoder shown in Fig. 2. (a) is formed by concatenation of two constituent codes in parallel and then by separation two codes by an interleaver. The recursive systematic convolutional code is usually used as a constituent code. The information bits are first encoded by a recursive systematic convolutional code, and then, after passing through an interleaver, are encoded by a second systematic convolutional encoder. The code sequences are formed by the information bits, followed by the parity check bits generated by both encoders. In Fig. 3. (a), the encoder takes data sequence $d_{k}$ as an input sequence and puts out three components: 1) $d_{k}$, information bits, 2) $x_{p 1, k}$, parity bit of the first encoder, and 3) $x_{p 2, k}$, parity bit of the second encoder.

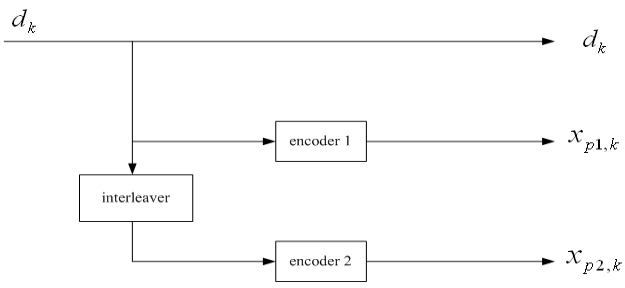

(a)

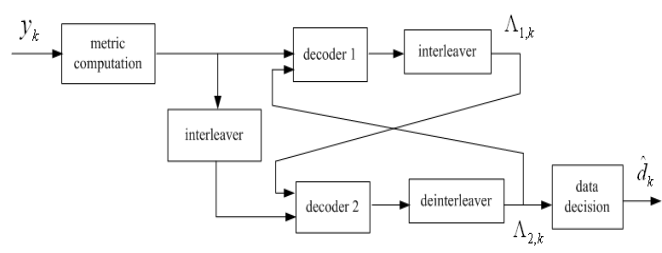

(b)

[Fig. 3] Block diagram of turbo encoder and decoder. (a) Turbo encoder. (b) Turbo decoder.

The most fundamental idea behind turbo code is interleaver gain by which error performance can be significantly improved through separation of constituent encoders. Thus, an appreciable increase in decoder complexity by increasing interleaver length results in substantial improvement of error performance. Unlike conventional channel coding schemes, turbo code need not trade off code rate or code complexity for increased Euclidean distance between codewords.

In the turbo decoder shown in Fig. 3. (b), the first thing to do is to compute metric. After that, the metric is used in the decoder 1 and decoder 2. The separate two decoders matched to the constituent encoders share soft 
reliability information in an iterative fashion. This soft reliability information (also called extrinsic information) is used as a priori information in the next decoding stage. Then, the performance of this iterative decoder becomes very close to that of ML (maximum likelihood) decoder with far less complexity. The soft outputs are derived from a modified Viterbi decoder, but the optimal soft output decoder is symbol by symbol APP (a posteriori probability) decoder whose outputs are the a posteriori probabilities of the decoded bits.

\section{Performance Analysis}

The OFDM system can be interpreted as a frequency multiplexing method for transmitting $K$ symbols simultaneously using $K$ subcarriers. The symbol sequence is divided into blocks of $K$ symbols. Then, the transmitted signal is given by

$$
\begin{aligned}
& x(t)=\sum_{k=0}^{K-1} x_{k} \exp \left(-j 2 k \pi f_{k} t-j 2 \pi f_{c} t\right), \\
& k T \leq t \leq(k+1) T,
\end{aligned}
$$

where $f_{c}$ is carrier frequency, $f_{k}$ is frequency of the k-th subcarrier, $T=T_{s}+T_{g}$ is sum of symbol duration ( $T_{s}$ ) and guard interval ( $T_{g}$ ), and $K$ is the number of subcarriers. At the receiver front end, the received signal is given by

$$
\begin{aligned}
& r(t)=\sum_{k=0}^{K-1} h_{k} x_{k} \exp \left(-j 2 k \pi f_{k} t-j 2 \pi f_{c} t\right), \\
& k T \leq t \leq(k+1) T,
\end{aligned}
$$

where $h_{k}$ is frequency response of the PLC channel at frequency $f_{c}+k f_{k}$. After sampling and taking FFT, the output signal of FFT algorithm is given by

$$
z_{k}=h_{k} x_{k}+n_{k}
$$

where $n_{k}$ is IFFT output of sampled noise. The bit error probability of 64 QAM with Gray mapping in an AWGN channel is given by [16]

$$
\begin{aligned}
& P_{b}=\frac{7}{24} \operatorname{erfc}\left(\sqrt{\frac{\gamma_{b}}{7}}\right)+\frac{1}{4} \operatorname{erfc}\left(3 \sqrt{\frac{\gamma_{b}}{7}}\right), \\
& -\frac{1}{24} \operatorname{erfc}\left(5 \sqrt{\frac{\gamma_{b}}{7}}\right)+\frac{1}{24} \operatorname{erfc}\left(9 \sqrt{\frac{\gamma_{b}}{7}}\right) \\
& -\frac{1}{24} \operatorname{erfc}\left(13 \sqrt{\frac{\gamma_{b}}{7}}\right)
\end{aligned}
$$

where

$$
\gamma_{b}=E_{b} / N_{0}
$$

$\operatorname{erfc}(x)=\frac{2}{\sqrt{\pi}} \int_{x}^{\infty} \exp \left(-u^{2}\right) d u$ is complementary error function.

For turbo coded codewords, codeword error probability is upper bounded by

$$
P_{w} \leq \sum_{d=1}^{N} A(d) P(d)
$$

where $N$ is block length of turbo codeword, $A(d)$ is the number of codewords with Hamming distance $d$, and $P(d)$ is decoding error probability of a codeword with weight $d$. To get the $A(d)$, we have to perform exhaustive search for a turbo code with fixed interleaver. So, by averaging over all possible interleavers, average weight distribution is obtained by

$$
A_{a}(d)=\sum_{i=1}^{Q}\left(\begin{array}{c}
Q \\
i
\end{array}\right) p(d \mid i)
$$

where $p(d i)$ is the probability that an input codeword with Hamming weight $i$ produces a codeword with Hamming weight $d$. The average upper bounds for word and bit error probabilities are, respectively, given by

$$
P_{w, a} \leq \sum_{d=d_{\min }}^{N} A_{a}(d) P(d)
$$




$$
P_{b, a}=\sum_{d=d_{\min }}^{N} \sum_{i=1}^{Q} \frac{i}{Q}\left(\begin{array}{c}
Q \\
i
\end{array}\right) p(d \mid i) P(d)
$$

where $d_{\min }$ is a minimum distance between codewords. To apply for the PLC channel, the turbo decoder should be modified to incorporate the PLC channel characteristics. For the turbo coded case, the bit error probability is evaluated through simulations using (12).

\section{Turbo Decoding Algorithms}

The turbo decoding algorithm depends on the available information on channel state in the decoder. The optimum decoding algorithms are based on MAP probability. The MAP algorithm has not been less popular than Viterbi algorithm because it achieves slightly better performance with higher complexity [17]. Recently, as a decoding algorithm of concatenated coding scheme, it is gathering spotlights with the increased attentions of turbo code. In a usual concatenated code, some kind of soft information is exchanged between the constituent codes.

In a concatenated code proposed by Forney, the optimum output of inner decoder becomes in the form of APP distribution [10]. In order to approach the required APP, many suboptimal algorithms have been considered. One of them is SOVA algorithm that is a kind of modified Viterbi algorithm compared to the original Viterbi algorithm. To make implementation easier, the Max Log Map algorithm has also been proposed. Since the optimal MAP decoding algorithm is very complex, so some suboptimal algorithms with less complexity have been proposed. In this paper, the Max Log MAP and the SOVA algorithms are considered as the suboptimal approaches.

\subsection{MAP Algorithm}

In the MAP algorithm, all the possible paths in the trellis are considered to optimally determine the reliability of information data. The MAP algorithm is computed through modified BCJR algorithm. Empirical evidences suggest that this decoding algorithm performs remarkably well and converges to the optimal decoding solution.
It can be noted that turbo decoding process can be regarded as the iterative improvement of APP because the $\log$ likelihood ratio is the ratio of APP of each information bit, $d_{k}=1$ and $d_{k}=0$. The turbo decoder makes full use of the APP (soft information) generated by the previous MAP decoder as a priori information. The APP is given by

$\Lambda\left(d_{k}\right)=\ln \frac{\sum_{S_{k}} \sum_{S_{k-1}} \gamma_{1}\left(y_{k}, S_{k-1}, S_{k}\right) \alpha_{k-1}\left(S_{k-1}\right) \beta_{k}\left(S_{k}\right)}{\sum_{S_{k}} \sum_{S_{k-1}} \gamma_{0}\left(y_{k}, S_{k-1}, S_{k}\right) \alpha_{k-1}\left(S_{k-1}\right) \beta_{k}\left(S_{k}\right)}$,

where $S_{k}$ is state of the first encoder at time $K$, and $a_{k}(\cdot)$ and $\beta_{k}(\cdot)$ are forward and backward recursion parameters, respectively.

The forward and backward recursion relations of the MAP are, respectively, given by

$$
\begin{gathered}
\alpha_{k}\left(S_{k}\right)=\frac{\sum_{S_{k}} \sum_{i=0}^{1} \gamma_{i}\left(y_{k}, S_{k-1}, S_{k}\right) \alpha_{k-1}\left(S_{k-1}\right)}{\sum_{S_{k}} \sum_{S_{k-1}} \sum_{i=0}^{1} \gamma_{i}\left(y_{k}, S_{k-1}, S_{k}\right) \alpha_{k-1}\left(S_{k-1}\right)}, \\
\beta_{k}\left(S_{k}\right)=\frac{\sum_{S_{k+1}} \sum_{i=0}^{1} \gamma_{i}\left(y_{k+1}, S_{k}, S_{k+1}\right) \beta_{k+1}\left(S_{k+1}\right)}{\sum_{S_{k}} \sum_{S_{k+1}} \sum_{i=0}^{1} \gamma_{i}\left(y_{k+1}, S_{k}, S_{k+1}\right) \alpha_{k}\left(S_{k}\right)}
\end{gathered}
$$

The initial conditions are $a_{0}\left(S_{0}\right)=0$ for $S_{0}=0$ and $\beta_{N}\left(S_{N}\right)=1$ for $S_{N}=0$. The branch transition probability of MAP decoder is given by

$$
\begin{aligned}
& \gamma_{i}\left(y_{k}^{s}, y_{k}^{p}, S_{k-1}, S_{k}\right)=q\left(d_{k}=i \mid S_{k}, S_{k-1}\right), \\
& \text { - } p\left(y_{k}^{s} \mid d_{k}-i\right) \cdot p\left(y_{k}^{p} \mid d_{k}=i, S_{k}, S_{k-1}\right) \\
& \text { - } p\left(S_{k} \mid S_{k-1}\right)
\end{aligned}
$$

where $z^{s}$ and $z^{p}$ are systematic and parity information of $z$, respectively, and $y_{k}^{s}$ and $y_{k}^{p}$ are 
channel outputs for $d_{k}$ and $x_{p 1, k}$, respectively. In (16), the value $q\left(d_{k}=i \mid S_{k}, S_{k-1}\right)$ is one for transition from $S_{k}$ to $S_{k-1}$, and zero for the other cases. The two outputs, $y_{k}^{s}$ and $y_{k}^{p}$, are used to compute a $\log$ likelihood ratio of a posteriori probabilities. This likelihood ratio is then employed to produce a priori probabilities in the following MAP decoder after which a new likelihood ratio is evaluated. This procedure is repeated many times until the bit error probability converges to some low value.

\subsection{Max-Log-MAP Algorithm}

In the Max Log MAP algorithm, the data decision and soft outputs are based on the best two paths with different distance. The Max Log Algorithm can be interpreted as a $\log$ domain version of the optimal MAP algorithm. To avoid complicated operations for $\gamma_{i}(\bullet, \bullet, \cdot), a(\bullet)$, and $\beta(\bullet)$, the logarithms of these values are taken in the Max Log MAP algorithm. Then, logarithmic branch transition probability is modified into

$$
\begin{aligned}
& \ln \gamma_{i}\left(\left(y_{k}^{s}, y_{k}^{p}\right), S_{k-1}, S_{k}\right)=\frac{2 y_{k}^{p} x_{k}^{p}(i)}{N_{o}} \\
& +\frac{2 y_{k}^{p} x_{k}^{p}\left(i, S_{k}, S_{k-1}\right)}{N_{o}}+\ln p\left(S_{k} \mid S_{K-1}\right)
\end{aligned}
$$

\subsection{SOVA Algorithm}

In the SOVA algorithm, two paths are considered, but the competing path may not be the best competing path. Usually, the competing path determines reliability of soft information and survives to merge with ML (maximum likelihood) path. In the conventional Viterbi algorithm, the path with the largest metric is selected as a survivor by maximizing, over all the possible paths, the value given by

$$
\sum_{k=1}^{N}\left[-\frac{1}{N_{0}}\left(y_{k}^{s}-x_{k}^{s}\right)^{2}-\frac{1}{N_{0}}\left(y_{k}^{p}-x_{k}^{p}\right)^{2}+\ln p\left(S_{k} \mid S_{k-1}\right)\right],
$$

In the hard decision, the Max Log MAP and SOVA algorithms work with the same metric. However, in the soft decision, these two algorithms operate differently. The SOVA algorithm computes only one competing path at every decoding step. For each information bit, it considers only a survivor of Viterbi algorithm.

\section{Simulation Results}

In this section, we present some simulation results. For simulation examples, the number of subcarriers $K=$ 1024 , transmission bandwidth $=8 \mathrm{MHz}$, interleaver length after serial to parallel converter $=512 \times 6$ symbols, guard interval $T_{g}=16 \mu_{S}$, FFT size $=1024$ are assumed. As a turbo scheme, the rate $1 / 3$ turbo code is used. And, as constituent codes, the recursive systematic convolutional codes are employed with code generator polynomials $(21,37)_{8}$ of octal representation.

The PLC system typically operates in a hostile noise environment. The channel impairments include background and non Gaussian noises such as impulse and harmonic noises. The background noise is modeled as AWGN (additive white gaussian noise) with two sided power spectral density (p.s.d.) of $N_{0} / 2$.

The harmonic noise is a periodic noise pulse that occur with a frequency other than multiples of the net voltage. The harmonic noise can be represented by

$$
n_{h}(t)=\sum_{k=1}^{N_{h}} A_{k} \cos \left(2 \pi k f_{0} t+k \ominus{ }_{0}\right)
$$

where $A_{k}$ is amplitude of the $k t h$ harmonic noise component, $f_{0}$ is fundamental frequency of the PLC, $N_{h}$ is the number of harmonic components, and $\Theta_{0}$ is random phase with uniform distribution in $(0,2 \pi)$. The harmonic noise is assumed to have mean which is 22 $\mathrm{dB}$ larger than the p.s.d. of background noise, and its variance and fundamental frequency are $6 \mathrm{~dB}$ and $60 \mathrm{~Hz}$, respectively. In the simulation, the number of harmonic components is assumed to be $N_{h}=3598$, and the 
largest harmonic frequency is calculated as $60 \mathrm{~Hz} \times 3598=215.88 \mathrm{kHz}$.

Random impulse noise can be caused by lightnings and load switching in the network (capacitor banks, thermostats, refrigerators, air conditioners, etc) with every impulse affecting a large frequency band. The impulses of the typical intrabuilding PLC channel have a period of approximately $8.3 \mathrm{msec}$ with equally likely impulse duration of approximately $40 \mu \mathrm{sec}$ or $80 \mu \mathrm{sec}$. The amplitudes of impulses are assumed to be 5 or 10 times larger than root mean square values of background noise and their probabilities of occurrences are approximately equal.

For the uncoded and the turbo coded case, the bit error probability in the PLC channel does not have a closed form. Therefore, as a numerical integration method, GQR (Gauss quadrature rule) is employed. The complementary error function is related by

$$
\operatorname{erfc}(x)=2 Q(\sqrt{2} x)
$$

where $Q(x)=\int_{x}^{\infty} \frac{1}{\sqrt{2 \pi}} \exp \left(-\frac{t^{2}}{2}\right) d t$ is tail integral of standard Gaussian density. For computation of $\operatorname{erfc}(x)$, the $Q$ - function (Gaussian tail integral) is used with the form given by

$$
Q(x)=\frac{1}{\pi} \int_{0}^{\pi / 2} \exp \left(-\frac{x^{2}}{2 \sin ^{2} \phi}\right) d \Phi
$$

In Fig. 4, for the optimal MAP decoding algorithm, bit error probability vs. SNR is compared for a different number of iterations. The simulation examples are shown for the impulse noise and interleaver length of turbo encoder $=1000$. It can be demonstrated that the turbo coding offers considerable coding gains as SNR increases compared with the uncoded case. It is confirmed that the turbo coding is very effective to improve performance of the OFDM/QAM system in the PLC channel. As the number of iterations increases, the performance is more improved through increased coding gain. However, it should be noted that the number of iterations exceeds some number (for this case, 8 ), the more iterations offers only marginal coding gain because the soft information is not available any longer after sufficient iterations.

In Fig. 5, for the optimal MAP decoding algorithm, bit error probability vs. SNR is compared for a different interleaver length of turbo encoder. The simulation examples are shown for the impulse noise and the number of iterations $=2$. The performance is significantly improved as the interleaver length of turbo encoder increases. However, increased interleaver length incurs increased complexity. Therefore, in a design of turbo encoder/decoder, trade off relationship in terms of performance and complexity should be taken into account.

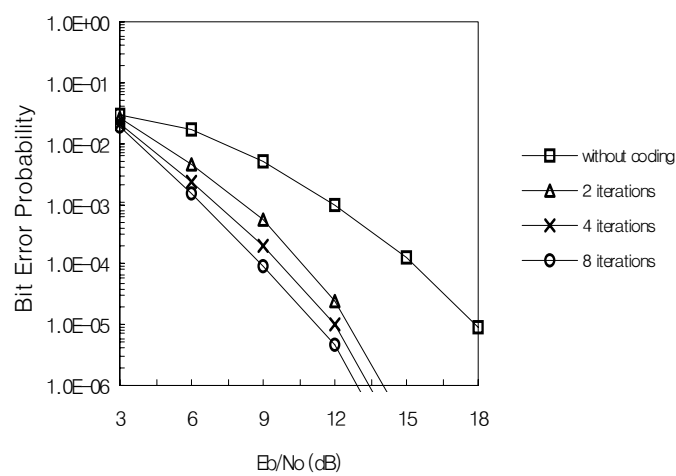

[Fig. 4] Bit error probability vs. SNR for a different number of iterations.

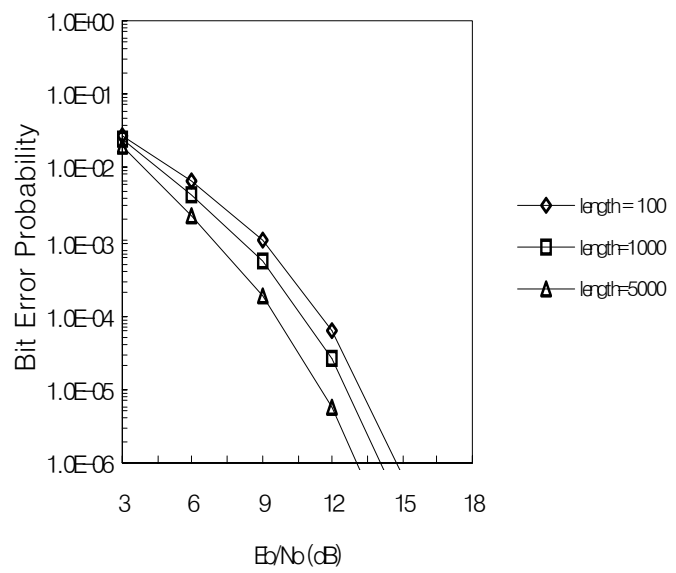

[Fig. 5] Bit error probability vs. SNR for a different interleaver length of turbo encoder. 


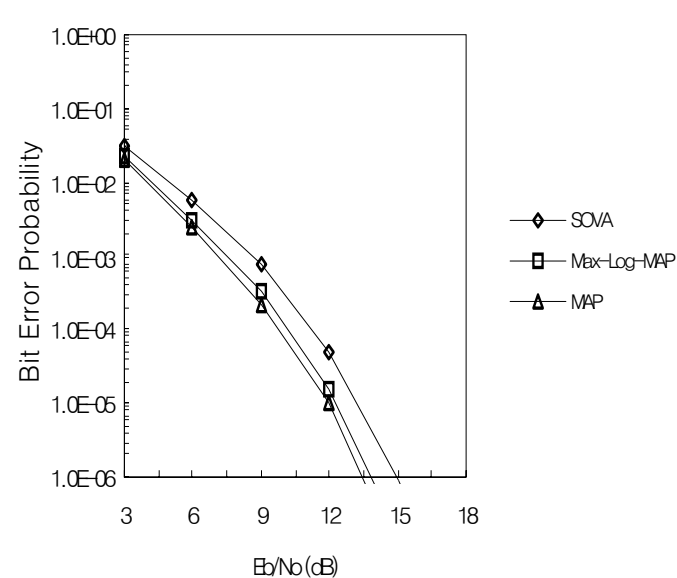

[Fig. 6] Bit error probability vs. SNR for different decoding algorithms.

In Fig. 6, bit error probability vs. SNR is compared for different decoding algorithms. The simulation examples are shown for the impulse noise, the number of iterations $=4$, and interleaver length of turbo encoder $=1000$. The optimal MAP algorithm achieves better performance compared with suboptimal algorithms such as Max Log

MAP and SOVA. However, the performance difference between the MAP and the Max Log MAP indicates only marginal difference. Therefore, it can be recommended that the Max Log MAP algorithm is better choice than the MAP algorithm in terms of both complexity and performance.

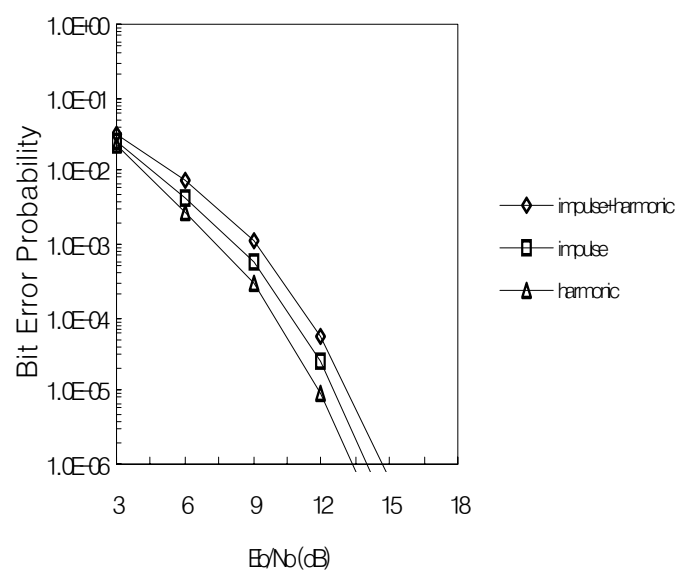

[Fig. 7] Bit error probability vs. SNR for various kinds of noise types.
In Fig. 7, for the optimal MAP decoding algorithm, bit error probability vs. SNR is compared for various kinds of noise types. The simulation examples are shown for the number of iterations $=2$ and interleaver length of turbo encoder $=1000$. The impact of impulse noise on BER performance is more critical than that of harmonic noise. From this figure, it is found that many kinds of noises substantially influence the performance of the PLC system.

\section{Conclusions}

The performance of a turbo coded OFDM/64 QAM system was analyzed and simulated in the PLC communication channel. The MAP, the Max Log MAP, and the SOVA algorithms were chosen and compared in the decoding process. It was confirmed that turbo coding provides considerable coding gains for the OFDM/QAM system in the PLC communication channel. From the simulation results, it was also demonstrated that the Max Log MAP algorithm is a suitable choice in terms of performance and complexity. It was confirmed that the performance is substantially improved by increasing the number of iterations and interleaver length of a turbo encoder.

These days, among the most challenging issues in the channel coding community is realization of turbo code with more reduced complexity while maintaining the near

optimal performance. Besides, in order to communicate reliably in the PLC channel, it takes a sophisticated modem since power lines basically entail high noise, high attenuation, and signal distortion. In realizing the high speed PLC modem, the following issues should be overcome; 1) impedance matching between loads, 2) noise reduction from home appliances, 3) time varying PLC channel, 4) limited spectrum, 5) wideband signal transmission, 6) electromagnetic radiation, etc.

It can be concluded that turbo coding is a very promising technique to enhance performance of OFDM system operating in the PLC communication channel. The results in this paper can be applied to OFDM based high speed PLC communication systems. 


\section{References}

[1] K. Dostert, Powerline Communications, Prentice Hall, 2001.

[2] M. L. Chan and R. W. Donaldson, "Attenuation of communication signals on residential and commercial intrabuilding power distribution circuits," IEEE Trans. Electromagn. Compat., vol. 28, no. 4, pp. 220 230, Nov. 1986

[3] M. L. Chan and R. W. Donalson, "Amplitude, width, and interarrival distributions for noise impulse on intrabuilding power line communication networks," IEEE Trans. Electormagn. Compat., vol. 31, no.4, pp. 320 323, Aug. 1989.

[4] O. Hooijen, "A channel model for the residential power circuit used as a digital communications medium," IEEE Trans. Electomagn. Compat, vol. 40, pp. 331 336, 1998.

[5] O. Hooijen, Aspects of Residential Power Line Communications, Shaker Verlag, Archen, Germany, 1998.

[6] C. Berrou, A. Glavieux, and P. Thitimajshima, "Near Shannon limit error correcting coding: turbo codes," in Proc. of IEEE ICC '93, pp. 1064 1070, Geneva, Switzerland, June 1993.

[7] S. Benedetto and G. Montorsi, "Unveiling turbo codes: Some results on parallel concatenated coding schemes," IEEE Trans. Inform. Theory, vol. 42, pp. 409 428, 1996.

[8] L. R. Bahl, J. Cocke, F. Jelinek, and J. Raviv, "Optimal decoding of linear codes for minimizing symbol error rates," IEEE Trans. Inform. Thoery, vol. 20, pp. 284 287, Mar. 1974.

[9] D. Divsalar, S. Dolinar, and F. Pollara, "Transfer function bounds on the performance of turbo codes," TDA Progress Report 42 123, Jet Propulsion Laboratory, Pasadena, CA, U.S.A, Aug. 1995.

[10] G. D. Forney, Jr., Concatenated Codes, MIT Press, 1966.

[11] S. B. Weinstein and P. M. Ebert, "Data transmission by frequency division multiplexing using the discrete Fourier transform," IEEE Trans. Commun., vol. 19, no. 5, pp. 628 634, Oct. 1971.

[12] J. A. C. Bingham, "Multicarrier modulation for data transmission: An idea whose time has come," IEEE Commun. Mag., vol. 28, no. 5, pp. 5 14, May 1990.

[13] L. J. Cimini, Jr., "Analysis and simulation of a digital mobile channel using orthogonal frequency division multiplexing," IEEE Trans. Commun., vol. 33, no. 7, pp. 665 675, July 1985.

[14] K. Dostert, "Power lines as high speed data transmission channels modeling the physical limits," Proc. of IEEE ISSSTA '98, Sun City, South Africa, Sept. 1998, pp. 585589.

[15] H. Philipps, "Modeling of powerline communication channels," Proc. of ISPLC '99, Lancaster, UK, Mar. 1999, pp. 1421

[16] M. K. Simon, S. M. Hinedi, and W. C. Lindsey, Digital Communication Techniques, Prentice Hall, 1995.

[17] J. Hagenauer and P. Hoeher, "A Viterbi algorithm with soft decision outputs and its applications," in Proc. of IEEE GLOBECOM '89, pp. 1680 1686, Dallas, TX, U.S.A, Nov. 1989.

Jin-Young Kim

[Regular member]

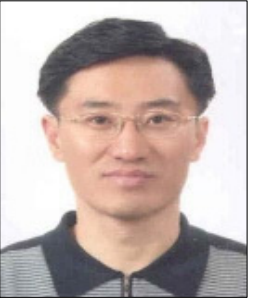

- Feb. 1998 : Seoul National University, $\mathrm{PhD}$

- Feb. $1998 \sim$ Feb. 1999 : Princeton University, Postdoctoral Research Fellow

- Mar. $2000 \sim$ Feb. 2001 : SK Telecom, Principal Member of Technical Staff

- Mar. $2001 \sim$ current : Kwangwoon University, Associate Professor

- Mar. $2009 \sim$ current : Massachusetts Institute of Technology (M.I.T), Visiting Scientist

$<$ Research Interests $>$

Spread-spectrum, Cognitive radio, Ultrawideband (UWB), Space communication, Optical communication and Powerline communication systems

Sung-Wan Koo

[Associate member]

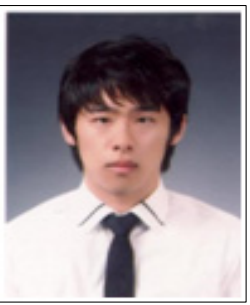

- Feb. 2008 : Kwangwoon University, Dep. of Wireless Communications Engineering, B.S

- Mar. $2008 \sim$ current : Kwangwoon University, Dep. of Wireless Communications Engineering, Master candidate

$<$ Research Interests $>$

Digital communication, Visible light communication, MIMO, Channel Coding, Cooperative communication, UWB 